\title{
Aneurisma de aorta abdominal: Importância do diagnóstico precoce
}

\author{
Abdominal aortic aneurysm: Importance of early diagnosis \\ Aneurisma de la aorta abdominal: Importancia del diagnóstico precoz
}

Lennara Pereira Mota

ORCID: https://orcid.org/0000-0002-2629-6634 Centro Universitário Maurício de Nassau, Brasil E-mail: lennaramota@hotmail.com

Luciano Paes Landim Ribeiro

ORCID: https://orcid.org/0000-0002-5495-7938

Centro Universitário Uninovafapi, Brasil

E-mail: lucym_srn@hotmail.com

Jaqueline Dantas da Costa

ORCID: https://orcid.org/0000-0003-2570-1949

Centro Universitário Uninovafapi, Brasil

E-mail: jaquedc87@gmail.com

Iaggo Henrique de Sousa Figueiredo

ORCID: https://orcid.org/0000-0001-6136-0411

Universidade Estadual do Piauí Brasil

E-mail: iaggo0106@hotmail.com

Douglas Bento das Chagas

ORCID: https://orcid.org/0000-0003-3141-8840

Fundação de Ensino Superior de Olinda, Brasil E-mail: douglasbchagas@hotmail.com

Mayara Rafaela dos Reis

ORCID: https://orcid.org/0000-0002-6973-004X

Centro Universitário Facid, Brasil

E-mail: mayara12reis@gmai.com

Maria Divina dos Santos Borges Farias

ORCID: https://orcid.org/0000-0002-1401-808X

Centro de Ensino Unificado de Teresina, Brasil

E-mail: mariadivina.bfarias@gmail.com

Tom Ravelly Mesquita Costa

ORCID: https://orcid.org/0000-0002-3092-6192

Universidade Federal do Delta do Parnaíba, Brasil

E-mail: tomravely20@hotmail.com

Rodrigo Araujo Belem

ORCID: https://orcid.org/0000-0003-2009-094X Instituto Tocantinense Presidente Antonio Carlos, Brasil E-mail: rodrigoaraujo115@gmail.com

Milena Matos da Cruz

ORCID: https://orcid.org/0000-0002-9875-8906 Instituto Tocantinense Presidente Antonio Carlos, Brasil

E-mail: milenamc_5@hotmail.com

Jose Antonio Rodrigues de Carvalho

ORCID: https://orcid.org/0000-0003-2009-094X Instituto Tocantinense Presidente Antonio Carlos, Brasil E-mail: joseantoniojlj@outlook.com

Amadeu Luis de Carvalho Neto ORCID: https://orcid.org/0000-0001-7726-6120 Universidade Federal do Piauí, Brasil E-mail: amadeusc.neto@hotmail.com

Millena Raimunda Martins de Almeida Carvalho ORCID: https://orcid.org/0000-0003-2431-2431 Universidade Federal do Piauí, Brasil

E-mail: millenamartinsalmeida2@gmail.com Wygor Bruno e Silva Morais

ORCID: https://orcid.org/0000-0001-5219-0465 Centro Universitário Unifacema, Brasil

E-mail:wygorleao@hotmail.com

Rômulo Henrique da Silva Lima ORCID: https://orcid.org/0000-0003-1715-3399 Secretaria Municipal De Barreirinhas, Brasil E-mail: romulo_gnr@hotmail.com 


\author{
Gersilane Lima Leal \\ ORCID: https://orcid.org/0000-0001-5272-1860 \\ Universidade Estadual do Piauí, Brasil \\ E-mail: gersilane.leal19@gmail.com \\ Eldson Rodrigues Borges \\ ORCID: https://orcid.org/0000-0002-8848-0074 \\ Centro Universitário Uninovafapi, Brasil \\ E-mail: eldsonborges7@gmail.com \\ Kelly Evenlly da Silva Santos \\ ORCID: https://orcid.org/0000-0002-6217-5143 \\ Centro Universitário Uninovafapi, Brasil \\ E-mail: kelly.evenlly@ hotmail.com \\ Fabiana Parente Macário da Silva \\ ORCID: https://orcid.org/0000-0002-1990-6406 \\ Centro Universitário Maurício de Nassau, Brasil \\ E-mail: fabianabiomedica01@gmail.com
}

\begin{abstract}
Resumo
As alterações na estrutura da aorta normal estão relacionadas ao desenvolvimento de doenças clínicas, como por exemplo, aneurisma da aorta abdominal. O aneurisma consiste em uma expansão anormal da aorta, que está localizada nos vasos sanguíneos e no coração e envolve três vestimentas externas. Na aorta, em comparação com a parte proximal, o diâmetro do vaso sanguíneo correspondente ao AAA aumenta cerca de 50\%. Como o AAA é na maioria das vezes assintomático e tem alta mortalidade por ruptura, o reconhecimento precoce e a correção cirúrgica eletiva são as melhores estratégias para prevenir o óbito. Sendo assim, esta revisão tem por objetivo expor através da análise de artigos científicos a importância do diagnóstico precoce de pacientes portadores de aneurisma de aorta abdominal. Trata-se de uma revisão bibliográfica de caráter qualitativo sistemático, realizada entre os anos de 2017 a 2021 nos idiomas português, inglês e espanhol. Alguns estudos recentes sobre a incidência de aneurismas da aorta abdominal parecem indicar que o progresso no tratamento desses pacientes está aumentando. A razão dessa evolução favorável deve ser atribuída às medidas de diagnóstico precoce dos pacientes em risco, fundamentalmente realizado por ultrassonografia. É necessária a utilização do diagnóstico precoce em pacientes que apresentem risco de desenvolver AAA, visto que o manejo cirúrgico eletivo tem permitido redução da mortalidade de forma considerável. Também são necessários estudos para o desenvolvimento de um tratamento farmacológico que se mostre eficaz em retardar a progressão e ruptura desses aneurismas.
\end{abstract}

Palavras-chave: Aorta abdominal; Dilatação; Fatores de risco; Diagnóstico.

\begin{abstract}
Changes in the structure of the normal aorta are related to the development of clinical diseases, such as abdominal aortic aneurysm. An aneurysm is an abnormal expansion of the aorta, which is located in the blood vessels and heart and involves three outer garments. In the aorta, compared to the proximal part, the diameter of the blood vessel corresponding to the AAA increases about 50\%. As AAA is most often asymptomatic and has high mortality due to rupture, early recognition and elective surgical correction are the best strategies to prevent death. Thus, this review aims to expose through the analysis of scientific articles the importance of early diagnosis of patients with abdominal aortic aneurysm. This is a systematic qualitative bibliographic review, carried out between 2017 and 2021 in Portuguese, English and Spanish. Some recent studies on the incidence of abdominal aortic aneurysms seem to indicate that progress in treating these patients is increasing. The reason for this favorable evolution must be attributed to the measures of early diagnosis of patients at risk, fundamentally performed by ultrasonography. It is necessary to use early diagnosis in patients who are at risk of developing AAA, since elective surgical management has allowed a considerable reduction in mortality. Studies are also needed to develop a pharmacological treatment that proves to be effective in delaying the progression and rupture of these aneurysms.
\end{abstract}

Keywords: Abdominal aorta; Dilation; Risk factors; Diagnosis.

\title{
Resumen
}

Los cambios en la estructura de la aorta normal están relacionados con el desarrollo de enfermedades clínicas, como el aneurisma de la aorta abdominal. Un aneurisma es una expansión anormal de la aorta, que se encuentra en los vasos sanguíneos y el corazón e involucra tres prendas externas. En la aorta, en comparación con la parte proximal, el diámetro del vaso sanguíneo correspondiente al AAA aumenta aproximadamente en un 50\%. Como el AAA suele ser asintomático y tiene una alta mortalidad debido a la rotura, el reconocimiento temprano y la corrección quirúrgica electiva son las mejores estrategias para prevenir la muerte. Así, esta revisión tiene como objetivo exponer a través del análisis de artículos científicos la importancia del diagnóstico precoz de los pacientes con aneurisma de aorta abdominal. Se trata de una revisión bibliográfica cualitativa sistemática, realizada entre 2017 y 2021 en portugués, inglés y español. Algunos estudios recientes sobre la incidencia de aneurismas de la aorta abdominal parecen indicar que los avances en el tratamiento de estos pacientes están aumentando. La razón de esta favorable evolución debe atribuirse a las medidas de diagnóstico precoz de los pacientes de riesgo, realizadas fundamentalmente por ecografía. Es necesario utilizar el diagnóstico precoz en pacientes con riesgo de desarrollar AAA, ya que el manejo quirúrgico 
electivo ha permitido una reducción considerable de la mortalidad. También se necesitan estudios para desarrollar un tratamiento farmacológico que demuestre ser eficaz para retrasar la progresión y rotura de estos aneurismas.

Palabras clave: Aorta abdominal; Dilatación; Factores de riesgo; Diagnóstico.

\section{Introdução}

Os aneurismas de aorta abdominal (AAA) são considerados um desafio terapêutico para o cirurgião vascular. A taxa de mortalidade é bastante alta, independentemente dos consideráveis avanços que ocorreram nas ultimas décadas com o surgimento, aprendizagem e validação de uma ampla gama de procedimentos endovasculares (Touma et al., 2021).

As alterações na estrutura da aorta normal estão relacionadas ao desenvolvimento de doenças clínicas, como por exemplo, aneurisma da aorta abdominal. O aneurisma consiste em uma expansão anormal da aorta, que está localizada nos vasos sanguíneos e no coração e envolve três vestimentas externas. Na aorta, em comparação com a parte proximal, o diâmetro do vaso sanguíneo correspondente ao AAA aumenta cerca de 50\%. Apresenta uma alta taxa de mortalidade quando rompida, algumas complicações como laceração e dissecção ocorrerão quando não houver tratamento, toda via a taxa de mortalidade é baixa quando é realizada a cirurgia eletiva (Santos et al., 2021).

Como principal artéria do nosso corpo, a aorta sai do coração, passa pelo tórax até o abdômen, onde se divide na artéria ilíaca comum, responsável pelo transporte do sangue para as extremidades inferiores. O aneurisma da aorta abdominal é uma protuberância causada pela expansão da parte inferior da aorta, podendo ocasionar um sangramento da parede vascular e levar a uma apoplexia, sendo o paciente diagnosticado clinicamente com hemoperitônio (Assis; Duque, 2020).

$\mathrm{O}$ aneurisma arterial (aórtico) é a expansão local e permanente dos vasos sanguíneos e é a causa da morte de muitos pacientes em todo o mundo. Os aneurismas da aorta podem causar complicações, como ruptura (quando um vaso sanguíneo se rompe) e, portanto, podem causar sangramento interno que requer intervenção cirúrgica para ser revertido (Menino et al., 2020).

A maioria dos pacientes não apresenta sintomas, portanto o diagnóstico pode ser uma descoberta inesperada, logo, deve-se começar pela investigação de grupos de alto risco para tratar a doença o mais precocemente possível. A evolução depende de vários fatores, dos quais o tamanho do aneurisma é um dos determinantes mais importantes do comportamento a ser seguido, pois constitui o maior fator prognóstico para o risco de ruptura (Guzmán et al., 2021).

O sintoma principal é dor abdominal aguda e intensa, podendo irradiar para os testículos, trato inguinal e/ou reto. Pode ocorrer também dor no quadril associado à irritação do músculo psoas (Touma et al., 2021).

$\mathrm{O}$ aneurisma da aorta abdominal pode ser determinado através dos diâmetros transversais e anteroposterior da aorta abdominal, sendo ambos maiores que $3,0 \mathrm{~cm}$. Geralmente é causada por alterações císticas na túnica média arterial, resultando em um aumento lento e contínuo do lúmen do vaso sanguíneo. A causa mais comum é a degeneração da parede arterial causada por doença aterosclerótica. Os principais fatores de risco para AAA são: tabagismo, hipertensão arterial sistêmica, idade avançada, raça branca, sexo masculino, história familiar positiva, dislipidemia, doença arterial coronariana e doença arterial periférica (Amaral et al., 2021).

A maioria dos pacientes com AAA é assintomática e é diagnosticada durante exames de imagem realizados para outros fins. $\mathrm{O}$ alto risco de ruptura e a taxa potencial de mortalidade estão intimamente relacionados ao tamanho e ao crescimento do aneurisma. Quando os sintomas aparecem, o diâmetro do aneurisma já atingiu cerca de $5 \mathrm{~cm}$, ou a expansão de mais de $1 \mathrm{~cm}$ em um ano, o tratamento cirúrgico é obrigatório. O tratamento seletivo do AAA pode ser realizado por duas técnicas distintas: cirurgia convencional aberta ou correção endovascular (Gomes et al., 2021).

A mortalidade dos indivíduos com aneurismas de aorta estão relacionadas significativamente com o rompimento do mesmo, provocando um quadro clínico de hemorragia no abdômen, sendo essencial uma correção cirúrgica de emergência para que o óbito seja evitado. Sendo assim, esse distúrbio vascular deve ser tratado de forma eletiva antes que ocorra a ruptura, com 
o objetivo de evitar tal complicação e diminuir a possibilidade de morte durante a cirurgia (Becker et al., 2020).

O estudo dos aneurismas é de bastante importância, visto que constituem uma causa considerável de morte súbita, pois quando ocorre o rompimento, podem provocar sangramento, às vezes tão forte, que leva a um estado de choque hipovolêmico, que leva ao óbito. O diagnóstico baseia-se no exame físico e métodos de diagnóstico por imagem (Ascanio et al., 2021).

Como o AAA é na maioria das vezes assintomático e tem alta mortalidade por ruptura, o reconhecimento precoce e a correção cirúrgica eletiva são as melhores estratégias para prevenir o óbito. Quando um aneurisma se rompe, apenas $25 \%$ dos pacientes chegam vivos ao hospital e apenas 10\% dos pacientes sobrevivem à cirurgia (Siqueira, 2021). Sendo assim, esta revisão tem por objetivo expor através da análise de artigos científicos a importância do diagnóstico precoce de pacientes portadores de aneurisma de aorta abdominal.

\section{Métodos}

Trata-se de uma revisão bibliográfica de caráter qualitativo sistemático. A busca pelos textos foi realizada a partir das seguintes palavras-chaves indexadas no DECS (Descritores em Ciências da Saúde): "Aorta abdominal"; "Dilatação"; "Fatores de risco"; "Diagnóstico", nos idiomas português, inglês e espanhol na plataforma Scientific Electronic Library Online (SCIELO) e Literatura Latino-Americana e do Caribe em Ciências da Saúde (LILACS).

Os critérios de inclusão foram pesquisas científicas publicadas de 2017 a 2021, publicados no idioma português, inglês e espanhol, que atendiam ao problema da pesquisa: Qual a importância do diagnóstico precoce em pacientes com Aneurisma de aorta abdominal? Os critérios de exclusão foram trabalhos científicos com apenas resumos disponíveis, publicações duplicadas, artigos de relato de experiência, reflexivo, editoriais, comentários e cartas ao editor.

A partir do problema de pesquisa foram selecionados artigos publicados em periódicos nacionais e internacionais escolhidos a partir de levantamento realizado por meios dos descritores na biblioteca virtual Scientific Electronic Library Online e Literatura Latino-Americana e do Caribe em Ciências da Saúde (LILACS) e dissertações e teses.

Os artigos foram selecionados para leitura e foram incluídos na analise qualitativa artigos que se enquadravam no tema proposto do trabalho.

\section{Resultados e Discussão}

A Figura 1 ilustra os resultados deste trabalho que foram obtidos através da analise qualitativa de publicações sobre a importância do diagnóstico precoce em pacientes portadores de aneurisma de aorta abdominal nas bases de dados Scientific Electronic Library Online e Literatura Latino-Americana e do Caribe em Ciências da Saúde (LILACS). 
Figura 1: Fluxograma que apresenta o processo de seleção das publicações sobre aneurisma de aorta abdominal e a importância do seu diagnóstico precoce, Teresina, Brasil, 2021.

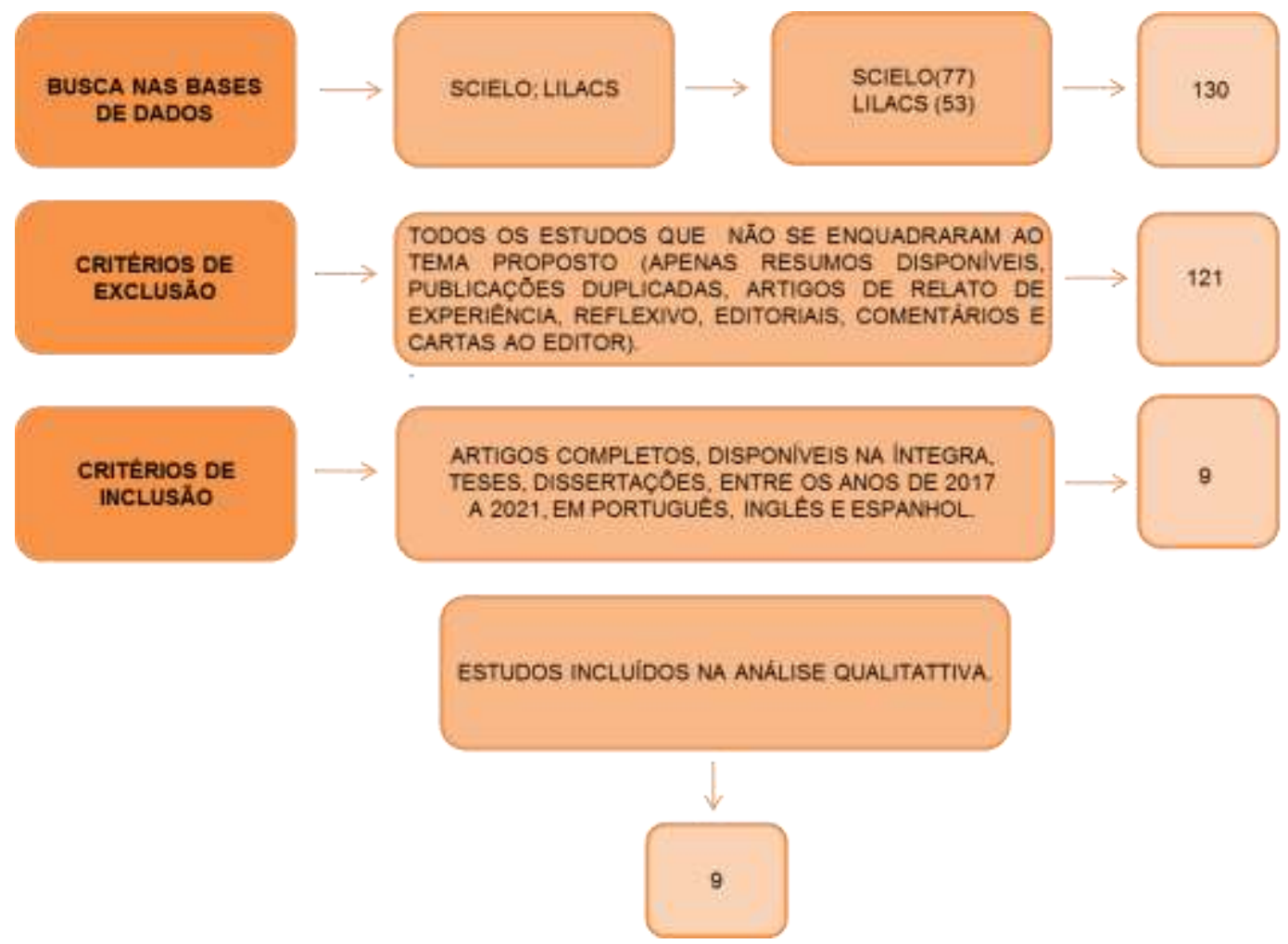

Fonte: (Mota et al., 2021).

Os resultados deste trabalho foram obtidos através da análise qualitativa de publicações sobre aneurisma de aorta abdominal e a importância do seu diagnóstico precoce encontrados nas bases de dados Scientific Electronic Library Online e Literatura Latino-Americana e do Caribe em Ciências da Saúde (LILACS) obtendo 77 artigos na SCIELO e 53 artigos no LILACS. Foram excluídos 72 artigos da SCIELO e 49 do LILACS por não se enquadrarem no tema proposto. Foram incluídos na análise qualitativa 05 artigos da SCIELO e 04 artigos da LILACS entre os anos de 2017 a 2021.

De acordo com a pesquisa realizada nas bases de dados, foi possivel listar os principais títulos e objetivos dos estudos publicados na literatura, utilizados neste trabalho conforme o Quadro 1.

Quadro 1. Principais títulos e objetivos dos artigos utilizados na pesquisa, Teresina, Brasil, 2021.

\begin{tabular}{|c|c|c|}
\hline ANO DE PUBLICAÇÃO & TÍTULO & OBJETIVO \\
\hline Rojas et al., 2021. & $\begin{array}{c}\text { Diagnóstico precoce de aneurisma } \\
\text { de aorta abdominal assintomático } \\
\text { em Pinar del Río. }\end{array}$ & $\begin{array}{c}\text { Caracterizar pacientes com diagnóstico de } \\
\text { aneurisma de aorta abdominal na província de } \\
\text { Pinar del Río. }\end{array}$ \\
\hline Guzmán et al., 2021. & $\begin{array}{c}\text { Complicações pós-cirúrgicas em } \\
\text { pacientes com aneurisma da aorta } \\
\text { abdominal operada eletivamente }\end{array}$ & $\begin{array}{c}\text { Caracterizar as principais complicações pós- } \\
\text { operatórias dos pacientes com aneurisma de } \\
\text { aorta abdominal operado eletivamente. }\end{array}$ \\
\hline Zayas et al., 2021. & $\begin{array}{c}\text { Reparo de aneurisma da aorta } \\
\text { torácica com comprometimento } \\
\text { Vasos do pescoço em um paciente } \\
\text { com sífilis terciária. Relato de caso. }\end{array}$ & $\begin{array}{c}\text { O presente caso clínico exemplifica a nova era } \\
\text { dos procedimentos invasivos híbridos na } \\
\text { resolução de patologias cardiovasculares em } \\
\text { busca de menor morbimortalidade por doenças, } \\
\text { rápida recuperação dos pacientes e sua } \\
\text { reintegração à vida produtiva em sociedade. }\end{array}$ \\
\hline Aneurisma de aorta abdominal: uma & Delinear o perfil epidemiológico dos pacientes \\
\hline
\end{tabular}




\begin{tabular}{|c|c|c|}
\hline Amaral; Fernandes; Alves, 2021. & $\begin{array}{c}\text { análise clínica- epidemiológica do } \\
\text { estado do Tocantins nos últimos 20 } \\
\text { anos. }\end{array}$ & $\begin{array}{c}\text { com aneurisma da aorta abdominal no estado } \\
\text { do Tocantins no período de 2000 a 2020. }\end{array}$ \\
\hline Silerio, 2021. & $\begin{array}{c}\text { Tratamento farmacológico de } \\
\text { aneurismas de aorta abdominal. }\end{array}$ & $\begin{array}{c}\text { Este artigo apresenta dados atualizados sobre } \\
\text { tratamentos farmacológicos que têm sido } \\
\text { estudados em ensaios clínicos e outras } \\
\text { estratégias terapêticas potenciais exploradas } \\
\text { em modelos animais. }\end{array}$ \\
\hline Trintinalha et al., 2021. & $\begin{array}{c}\text { Derivação axilo-ilíaco para proteção } \\
\text { de rim transplantado durante a } \\
\text { correção de aneurisma de aorta } \\
\text { abdominal: relato de caso. }\end{array}$ & $\begin{array}{c}\text { O objetivo deste estudo foi relatar a abordagem } \\
\text { de um caso de AAA em um paciente } \\
\text { transplantado renal. O protocolo foi aprovado } \\
\text { pelo Comitê de Ética da nossa instituição } \\
\text { (parecer número 4.192.559). }\end{array}$ \\
\hline Gomes; D’Ornellas; Dotto, 2021. & $\begin{array}{c}\text { Medidas de aneurisma da aorta } \\
\text { abdominal: modelos tridimensionais } \\
\text { impressos e virtuais. }\end{array}$ & $\begin{array}{c}\text { Validar a aplicação do método de impressão } \\
\text { tridimensional de biomodelos para aferição } \\
\text { métrica e volumétrica de segmentos de } \\
\text { aneurisma de aorta abdominal. }\end{array}$ \\
\hline Santos et al., 2021. & $\begin{array}{c}\text { Podemos adotar a dosagem sérica da } \\
\text { Tenascina-C na avaliação } \\
\text { prognóstica do aneurisma e } \\
\text { dissecção da aorta? }\end{array}$ & $\begin{array}{c}\text { O objetivo desta revisão foi realizar um } \\
\text { levantamento na literatura com o propósito de } \\
\text { avaliar se a TN-C pode auxiliar na avaliação do } \\
\text { prognóstico do AAA e da DAA. }\end{array}$ \\
\hline Rilva et al., 2020. & $\begin{array}{c}\text { Relação morfológica entre o } \\
\text { diâmetro, o colo proximal e distal } \\
\text { dos aneurismas da aorta abdominal. }\end{array}$ & $\begin{array}{c}\text { Analisar relevantes aspectos anatômico- } \\
\text { morfológicos dos aneurismas da aorta } \\
\text { abdominal encontrados em necropsia. }\end{array}$ \\
\hline
\end{tabular}

Fonte: SCIELO e LILACS.

Desta forma, foi possível listar os principais resultados e conclusões dos estudos publicados na literatura, conforme o

Quadro 2.

Quadro 2. Resultados e conclusões dos artigos utilizados, Teresina, Brasil, 2021.

\begin{tabular}{|c|c|c|}
\hline PUBLICAÇÃO & RESULTADOS & CONCLUSÕES \\
\hline Rojas et al., 2021. & $\begin{array}{l}58,56 \% \text { dos pacientes foram identificados pelo } \\
\text { serviço de imagem. Predominou o sexo } \\
\text { masculino }(95,7 \%) \text {, sendo tabagistas }(90 \%) \text { e } \\
\text { hipertensos }(85,7 \%) \text { fatores de risco. O } \\
\text { crescimento do saco aneurismático foi } \\
\text { observado em relação à hipertensão arterial; } \\
\text { não houve aumento do diâmetro em } 44,3 \% \text { dos } \\
\text { pacientes. } 7,15 \% \text { foram operados, sendo } 100 \% \\
\text { pacientes com diâmetro inicial superior a } 5 \mathrm{~cm} \text {. }\end{array}$ & $\begin{array}{l}\text { Aneurismas de aorta abdominal foram } \\
\text { comuns em pacientes do sexo masculino } \\
\text { após a quarta década de vida, } \\
\text { principalmente em fumantes e hipertensos. } \\
\text { O departamento de radiologia foi a } \\
\text { principal fonte de encaminhamento. A } \\
\text { identificação precoce e o tratamento } \\
\text { oportuno são elementos determinantes para } \\
\text { um desfecho positivo desta doença. }\end{array}$ \\
\hline Guzmán et al., 2021. & $\begin{array}{l}56,4 \% \text { dos pacientes operados apresentavam } \\
\text { algum tipo de complicações, que foram mais } \\
\text { frequentes em homens com mais de } 60 \text { anos. As } \\
\text { mais prevalentes foram broncopneumonia } \\
(24,5 \%) \text { e arritmias cardíacas }(20,8 \%) \text {. A } \\
\text { proporção de complicações foi maior em } \\
\text { pacientes com enxertos bifurcados, bem como } \\
\text { aqueles sem profilaxia e com tempo cirúrgico } \\
\text { prolongado. Houve maior frequência de } \\
\text { doenças cardiovasculares entre os falecidos. }\end{array}$ & $\begin{array}{l}\text { As complicações respiratórias e } \\
\text { cardiovasculares predominaram nos } \\
\text { pacientes operados eletivamente para } \\
\text { aneurisma de aorta abdominal, este último } \\
\text { causando aumento da mortalidade. }\end{array}$ \\
\hline Zayas et al., 2021. & $\begin{array}{c}\text { Uma limitação foi não haver estudo } \\
\text { anatomopatológico confirmatório das afecções } \\
\text { microvasculares típicas da sífilis terciária, pois } \\
\text { o procedimento exigia ritmo vertiginoso devido } \\
\text { ao comprometimento hemodinâmico do } \\
\text { paciente. O sucesso foi, sem dúvida, devido à } \\
\text { abordagem multidisciplinar que foi feita no } \\
\text { caso. }\end{array}$ & $\begin{array}{l}\text { A abordagem endovascular para o reparo de } \\
\text { patologias da aorta nos últimos anos tem } \\
\text { evoluído a ponto de proporcionar uma } \\
\text { melhor relação de morbimortalidade em } \\
\text { relação às técnicas cirúrgicas } \\
\text { convencionais, ideais para países em } \\
\text { desenvolvimento por reduzir } \\
\text { significativamente os tempos de } \\
\text { recuperação e consequentemente } \\
\text { hospitalização. }\end{array}$ \\
\hline
\end{tabular}




\begin{tabular}{|c|c|c|}
\hline $\begin{array}{l}\text { Amaral; Fernandes; Alves, } \\
2021 .\end{array}$ & $\begin{array}{l}\text { Quando as manifestações clínicas estão } \\
\text { presentes, podem incluir sintomas vagos e } \\
\text { inespecíficos, como dor abdominal difusa, } \\
\text { dores no dorso e região supraumbilical, ou nos } \\
\text { flancos com irradiação para o dorso, virilha ou } \\
\text { escroto. Esses sintomas podem resultar da } \\
\text { pressão direta provocada pelo aneurisma ou } \\
\text { distensão dos músculos intra-abdominais e das } \\
\text { estruturas adjacentes à aorta. }\end{array}$ & $\begin{array}{l}\text { A escassez de estudos sobre o AAA no } \\
\text { estado do Tocantins evidencia a } \\
\text { necessidade da realização de programas de } \\
\text { rastreio populacional, com o objetivo de } \\
\text { diagnosticar precocemente o AAA, para } \\
\text { que manejo terapêutico correto possa ser } \\
\text { estabelecido de maneira eletiva, reduzindo, } \\
\text { assim, suas possíveis complicações. }\end{array}$ \\
\hline Silerio, 2021. & $\begin{array}{l}\text { Em um estudo recente que avaliou o efeito } \\
\text { desta droga na prevenção da desestabilização e } \\
\text { ruptura da placa aterosclerótica, verificou-se } \\
\text { que a administração intravenosa semanal de } \\
\text { pioglitazona-np ( } 7 \mathrm{mg} / \mathrm{kg} \text { por semana) por } 4 \\
\text { semanas, o risco de interrompendo. Este efeito } \\
\text { é devido ao fato de que a pioglitazona-np } \\
\text { regulou a expressão de citocinas inflamatórias } \\
\text { em macrófagos derivados da medula óssea e } \\
\text { suprimiram a expressão do indutor de mmp. }\end{array}$ & $\begin{array}{c}\text { Existem vários estudos em que os } \\
\text { benefícios de diferentes medicamentos } \\
\text { foram avaliados; No entanto, nenhum deles } \\
\text { apresenta evidências suficientes para } \\
\text { justificar sua introdução no gestão } \\
\text { terapêutica da aaa, para a qual é necessária } \\
\text { a realização de ensaios clínicos de maior } \\
\text { qualidade, devido aos resultados } \\
\text { discrepantes encontrados na utilização de } \\
\text { ieca, tetraciclinas e antiagregantes } \\
\text { plaquetários. }\end{array}$ \\
\hline Trintinalha et al., 2021. & $\begin{array}{l}\text { Recomenda-se o reparo imediato de todos os } \\
\text { AAA com diâmetro igual ou superior a } 5,0 \mathrm{~cm} \\
\text { e vigilância rigorosa (imagens a cada } 6 \text { meses) } \\
\text { para os aneurismas com menor diâmetro. } \\
\text { Comparados aos pacientes sem transplante, os } \\
\text { aneurismas da aorta em pacientes com } \\
\text { transplantes de órgãos abdominais e cardíacos } \\
\text { se expandem duas vezes mais rapidamente. }\end{array}$ & $\begin{array}{c}\text { A cirurgia convencional aberta com } \\
\text { derivação extra anatômica temporária é } \\
\text { uma alternativa para o tratamento do AAA } \\
\text { em pacientes com transplante renal. }\end{array}$ \\
\hline $\begin{array}{c}\text { Gomes; D’Ornellas; Dotto, } \\
2021 .\end{array}$ & $\begin{array}{c}\text { A avaliação da volumetria demonstrou } \\
\text { discrepância de } 3 \% \text { a } 12 \% \text { e a diferença entre as } \\
\text { áreas foi de } 10 \% \text { a } 16 \% \text {. }\end{array}$ & $\begin{array}{l}\text { A tomografia computadorizada dos } \\
\text { biomodelos impressos é compatível nas } \\
\text { aferições métricas e volumétricas com as } \\
\text { imagens tridimensionais da } \\
\text { angiotomografia do paciente. }\end{array}$ \\
\hline Santos et al., 2021. & $\begin{array}{l}\text { Constatou-se correlação negativa entre níveis } \\
\text { séricos de TN-C e a variação do diâmetro da } \\
\text { aorta. A partir dessa correlação negativa, os } \\
\text { autores concluíram que níveis séricos elevados } \\
\text { de TN-C no sétimo dia após admissão } \\
\text { hospitalar pode não apenas predizer a regressão } \\
\text { da DAA, como também induzir um efeito } \\
\text { protetor em relação ao alargamento da lesão } \\
\text { aórtica durante o desenvolvimento do estágio } \\
\text { crônico da doença. }\end{array}$ & $\begin{array}{c}\text { A utilização prática da TN-C ainda carece } \\
\text { de mais esclarecimentos devido a uma série } \\
\text { de limitações. Mais pesquisas precisam ser } \\
\text { realizadas para que essa glicoproteína seja } \\
\text { utilizada amplamente. Um ponto que } \\
\text { permanece sem uma resposta definitiva, } \\
\text { porém, reside na interpretação dos níveis } \\
\text { séricos elevados de TN-C }\end{array}$ \\
\hline Da Silva et al., 2020. & $\begin{array}{l}\text { A média do valor do diâmetro transverso na } \\
\text { amostra foi de } 6,86 \mathrm{~cm} \text { e a média da extensão } \\
\text { longitudinal, } 10,03 \mathrm{~cm} \text {. Trinta e nove } \\
\text { aneurismas apresentavam colo proximal com } \\
\text { diâmetro médio de } 2,33 \mathrm{~cm}(1,9 \text { a } 2,8 \mathrm{~cm}) \text { e } \\
\text { comprimento variando de } 0,4 \text { a } 6,8 \mathrm{~cm} \text { (média } \\
\text { de } 2,41 \mathrm{~cm} \text { ). Em } 12 \text { espécimes, encontrou-se } \\
\text { colo distal (média do diâmetro de } 2,44 \mathrm{~cm} \text {, } \\
\text { média do comprimento de } 1,58 \mathrm{~cm} \text { ). }\end{array}$ & $\begin{array}{l}\text { Nesta amostra, os aneurismas com maior } \\
\text { diâmetro e comprimento tendem a } \\
\text { apresentar menor comprimento do colo } \\
\text { proximal e distal. Esse fato demonstra que à } \\
\text { medida que o aneurisma cresce, ele perde } \\
\text { os colos anatômicos e avança em direção às } \\
\text { artérias ilíacas e renais. A progressão é } \\
\text { mais acentuada em sentido distal, pois a } \\
\text { minoria dos espécimes tem colo anatômico } \\
\text { antes da bifurcação da aorta. }\end{array}$ \\
\hline
\end{tabular}

\section{Fonte: SCIELO e LILACS.}

De acordo com os artigos científicos encontrados nota-se que o aneurisma da aorta abdominal é um distúrbio vascular com alta morbimortalidade, podendo chegar a $8 \%$ em homens com mais de 65 anos. Nessa doença, geralmente assintomática, as paredes dos vasos sanguíneos se expandem progressivamente, o que pode causar sua ruptura, o que é um fenômeno fatal em 
mais de $80 \%$ dos casos. O tratamento de pacientes com aneurisma assintomático é limitado a exames de imagem de acompanhamento regular, controle de fatores de risco cardiovascular, terapia antiplaquetária e terapia com estatinas. No entanto, não existe atualmente nenhum tratamento medicamentoso eficaz que possa limitar seu progresso ou prevenir sua ruptura. Atualmente, o diâmetro da aorta é o principal indicador do risco de rompimento, quando atinge um valor superior a $5,5 \mathrm{~cm}$, determina-se a necessidade ou não de correção cirúrgica (Rojas et al., 2021).

A estrutura do tecido da aorta é formada por camadas chamadas de túnicas, que são divididas em: adventícia, média e íntima. São compostos por células musculares, elastina, colágeno, células endoteliais e glicosaminoglicanos, estes últimos com a função de impedir a aderência das células sanguíneas à parede do vaso (De Assis; Duque, 2020).

Por meio da análise histológica, podemos entender a patogênese do AAA. A destruição da camada intermediária e a perda da estrutura fibrosa do colágeno fazem parte desta patogênese. A estrutura fibrosa do colágeno é substituída pela matriz extracelular (MEC), o que resulta em tecidos frágeis e inelásticos que são propensos à expansão progressiva da parede. Elastase e metaloproteinases (mmp) são alguns dos principais fatores bioquímicos envolvidos, assim como macrófagos e citocinas, o que indica que a inflamação é um componente participante da fisiopatologia (Silerio, 2021).

$\mathrm{O}$ aneurisma de aorta abdominal é o mais comum, mesmo quando comparado a outras partes da aorta. Um aneurisma é o resultado da degeneração da camada média da artéria, fazendo com que o lúmen vascular se expanda lenta e continuamente. Apesar da contínua pesquisa técnico-científica sobre a formação do aneurisma, ele ainda não está diretamente relacionado à doença aterosclerótica, que costuma ser considerada a principal causa desse processo. Outras causas incluem trauma, infecção, artrite, necrose cística da túnica média, doenças congênitas do tecido conjuntivo, como síndrome de Marfan e ruptura anastomótica (Gomes et al., 2021; Trintinalha et al., 2021).

A aorta é a principal artéria do corpo, em consequência do seu alto calibre e da sua grande importância para o fluxo do sangue. Ela emerge do coração a partir da sua porção ascendente e seu diâmetro varia de 2 a $3,0 \mathrm{~cm}$. É dividida em quatro porções: parte ascendente da aorta, o arco da aorta, parte torácica da aorta e a parte abdominal da aorta. Os aneurismas da aorta abdominal afetam frequentemente o segmento da aorta localizado entre as artérias renais e a artéria mesentérica inferior (Amaral et al., 2021).

Apesar dos sinais envolvidos no aneurisma de aorta abdominal, ele assintomático, frequentemente quando o paciente vem a sentir alguns sintomas como: dor lombar e/ou sensação de massa pulsátil na região do abdômen, o aneurisma já está muito dilatado ou próximo do rompimento (Assis; Duque, 2020).

O exame físico indica palidez, transpiração excessiva, e eventualmente, presença de uma massa abdominal pulsátil, sendo visível em pacientes magros. Raramente, podem ser observadas equimoses dos flancos, em consequência da difusão do sangue nas rupturas retroperitoneais. Em situações como, de choque hemorrágico, é observado livedo reticular. Quando é realizado à palpação, sente-se uma massa abdominal dolorosa e expansiva, sendo mais difícil ou impossível de notar em pacientes obesos, bem como um pulso rápido e filiforme, podendo ser até ausente em caso de choque (Touma et al., 2021).

Quando o diâmetro do vaso sanguíneo atinge ou ultrapassa 50\% do diâmetro normal da aorta, um aneurisma aórtico deve ser considerado. As causas do AAA são muitas, mas a maioria delas se deve à deterioração da parede dos vasos sanguíneos causada pelo processo inflamatório crônico e, na maioria dos casos, é causada pelo acúmulo de gordura. Com o tempo, a degeneração da parede aórtica a enfraquece, e a força exercida pela pressão arterial pode causar ruptura, chamada de dissecção do aneurisma, causando choque hipovolêmico, que é a principal causa de morte em pacientes com AAA (De Assis; Duque, 2020).

O AAA é definido por alterações na estrutura da parede da artéria, que demonstram o seu enfraquecimento. Sua história natural deve-se ao fato de que o crescimento contínuo pode levar a ruptura do vaso, sendo uma manifestação clínica muito temida, e que está associada a uma alta taxa de mortalidade, cerca de 80 a $90 \%$ (García et al., 2021). 
Há evidências de que a patogênese do AAA é multifatorial, e fatores genéticos e ambientais contribuem para o desenvolvimento da doença. A maioria dos AAAs tem causas degenerativas. A fisiopatologia envolvida em sua formação não foi totalmente elucidada, mas sabe-se que envolve muitos fatores, como vias metabólicas proteolíticas, inflamação, estresse oxidativo e degeneração da matriz extracelular (Siqueira, 2021).

O cuidado de pacientes com aneurisma é bastante complexo, porque os aneurismas apresentam diferentes riscos de rompimento e os fatores específicos de cada paciente podem afetar diretamente na expectativa de vida, nos riscos nos procedimentos cirúrgicos e das necessidades de intervenção. Decidir qual estratégia cirúrgica utilizar associada ao melhor tratamento de comorbidades pré-existentes é de fundamental importância para alcançar excelentes resultados. Além disso, o monitoramento pós-operatório adequado é necessário para minimizar as taxas de óbito ou morbidade subsequente associada ao aneurisma (Novais et al., 2021).

A aorta é classificada anatomicamente e utiliza como parâmetro a sua relação com o diafragma torácico-abdominal. As características estruturais dos aneurismas podem ser classificadas de forma macroscópica em verdadeiras e falsas, conforme as camadas do vaso envolvido, as verdadeiras são consideradas aquelas em que a dilatação afeta as três camadas e as falsas (pseudoaneurisma) são aquelas em que o comprometimento corresponde a uma das três camadas. Outra classificação baseia-se em sua forma, onde temos os saculares em que apenas um segmento curto da artéria é afetado e os fusiformes onde há um extenso comprometimento da artéria (Zayas et al., 2021).

Clinicamente, antes de um evento de ruptura catastrófico, a maioria dos aneurismas da aorta abdominal costuma estar silencioso. O AAA geralmente é encontrado acidentalmente durante os testes de diagnóstico por imagem usados para outros fins. Um aneurisma de aorta abdominal rompido se manifesta como uma tríade de dor abdominal ou dor lombar, choque hipovolêmico e massa abdominal pulsátil. O principal risco associado aos aneurismas é a ruptura, que é um evento com alto índice de letalidade (Amaral et al., 2021).

O aneurisma de aorta define-se como uma dilatação focal de uma artéria, que representa um aumento de mais de 50\% do diâmetro esperado, sendo baseado em medidas médias obtidas em um estudo de tomografia axial computadorizada (TC) realizado na população geral. No caso da aorta abdominal, corresponde a um diâmetro superior a $3 \mathrm{~cm}$. Afeta cerca de $4 \%$ dos homens acima de 60 anos e sua localização geralmente é infrarenal. A complicação mais comum do aneurisma de aorta abdominal é a ruptura espontânea, com uma taxa de mortalidade entre 50\% a 80\%. Em contrapartida, a taxa de mortalidade cirúrgica programada para aneurismas não complicados em pacientes de baixo risco é próxima a $2 \%$. Esses dados apontam que o tratamento é necessário antes da ruptura, e a comunidade médica geralmente concorda que AAAs com diâmetro superior a 5 $\mathrm{cm}$ devem ser tratados. O tratamento tradicional é a laparotomia, com exceção de pacientes de alto risco, com mortalidade em 30 dias próxima a 8\% (Rojas et al., 2021).

O tempo decorrido entre o diagnóstico e o procedimento cirúrgico tem influência na sobrevida dos pacientes. A técnica cirúrgica pode variar, levando em consideração diversos fatores como, por exemplo, a idade do paciente, a localização e extensão do aneurisma, o diâmetro do vaso, as condições hemodinâmicas e o centro de saúde onde o reparo cirúrgico será realizado. A idade está evidentemente associada ao risco do desenvolvimento de AAA, embora a morte por ruptura seja improvável em pacientes abaixo dos 55 anos de idade. Essa ligação com a idade avançada está relacionada ao aumento dos fatores de risco cardiovasculares relacionados a essa patologia (Ascanio et al., 2021).

Mais de meio século se passou desde que o reparo aberto do aneurisma da aorta abdominal foi retratado pela primeira vez. Desde então, seu tratamento cirúrgico teve avanços importantes. O AAA é uma patologia gravíssima com complicações fatais e sua mortalidade cirúrgica está entre 30\% a 63\%. Ocupa posição de destaque na população acima de 60 anos, sendo que 10 em cada 100 pessoas sofrem com essa doença. A frequência está aumentando porque qualquer condição patológica que enfraqueça a parede arterial a tornará propensa à formação de aneurisma. Nos Estados Unidos, cerca de 9\% da população com 
mais de 65 anos são diagnosticados com AAA e, devido à sua ruptura, em torno de 15.000 pessoas morrem anualmente. 1 em cada 3 pacientes chega com vida ao hospital. Cerca de $70 \%$ dos aneurismas de aorta estão localizados no abdômen e apresenta forte tendência ao rompimento, o que está diretamente associado ao tamanho e forma. Devido à sua importância no prognóstico e tratamento, sua localização deve ser considerada. A parte inferior do rim está envolvida principalmente, apenas $5 \%$ da aorta adrenal está envolvida e a artéria ilíaca está envolvida em $25 \%$ dos casos. A patogênese é complicada e não é bem esclarecida. Este distúrbio é de origem genética, mas refere-se especificamente a arteriosclerose e doença aórtica degenerativa, embora outras causas também sejam reconhecidas (Guzmán et al., 2021).

Alguns estudos recentes sobre a incidência de aneurismas da aorta abdominal parecem indicar que o progresso no tratamento desses pacientes está aumentando. A razão dessa evolução favorável deve ser atribuída às medidas de diagnóstico precoce dos pacientes em risco, fundamentalmente realizado por ultrassonografia. A perspectiva de novos casos no futuro depende de duas circunstâncias: por um lado, a população em geral está mais envelhecida, por outro, a possibilidade de detecção de novos casos de aneurisma na população (Rojas et al., 2021).

Geralmente, a ultrassonografia abdominal é considerada o método de rastreamento preferido para AAA devido à sua alta sensibilidade e especificidade, segurança e custo relativamente baixo. O aneurisma de aorta abdominal é frequentemente encontrado de forma acidental, especialmente em pacientes idosos, sendo responsável por 90 a $95 \%$ de todos os casos de aneurisma da aorta. Estima-se que a prevalência de AAA na população de 60 anos seja de $2 \%$ e cerca de $5 \%$ após os 70 anos. A incidência em homens é 2 a 3 vezes maior que nas mulheres (Amaral et al., 2021).

O método perfeito para a confirmação de AAA é a ultrassonografia abdominal, pois é considerada 95\% confiável, e possui alta sensibilidade e especificidade. É uma técnica não invasiva, sem apresentação de complicações físicas ou psicológicas e pode ser executada em regime ambulatorial (Ascanio et al., 2021).

A angiotomografia de aorta abdominal é utilizada no planejamento do procedimento cirúrgico, para detecção do tipo, localização, tamanho, acometimento dos ramos da aorta e presença de trombos e calcificações dentro do aneurisma. A tortuosidade, angulação e extensão do trombo intraluminal devem ser analisadas, pois apresentam influencia no risco de embolização durante o procedimento (Gomes et al., 2021).

Sabe-se que a cirurgia do aneurisma da aorta abdominal é uma cirurgia considerada de alto risco, com maior incidência de óbitos em idosos. O desenvolvimento de AAA envolve aumento do diâmetro normal e ruptura esporádica e tem afetado mais e mais pessoas nos últimos 30 anos. A idade e presença de doença crônica são fatores que devem ser considerados quando pacientes com AAA recebem tratamento de aneurisma, visto que esses indivíduos pertencem ao grupo de alto risco para o procedimento cirúrgico (Menino et al., 2020).

O tratamento do aneurisma da aorta abdominal é cirúrgico e as técnicas convencionais incluem a substituição do aneurisma dilatado por uma prótese vascular. O tratamento cirúrgico tem sido amplamente aceito e estabeleceu resultados já definidos e aceitáveis em longo prazo, para pacientes que apresentam alto risco de rompimento. Esses pacientes apresentam geralmente diâmetro maior que $5 \mathrm{~cm}$, hipertensão diastólica e doença pulmonar obstrutiva com VEF1 menor que $50 \%$ (Becker et al., 2020).

Com o advento das técnicas de reparo intra-arterial dos aneurismas da aorta abdominal, as características anatômicas desses aneurismas tornaram-se mais importantes. Dependendo do diâmetro do aneurisma, o colo proximal, o colo distal, o grau de tortuosidade e o grau de envolvimento dos vasos ilíacos terão variações. Portanto, é importante saber se o crescimento vai complicar o aneurisma, dificultando o tratamento intervencionista. As características anatômicas do aneurisma são obtidas por tomografia computadorizada convencional relacionada à angiografia, tomografia computadorizada espiral ou angiografia por ressonância magnética com gadolínio antes da cirurgia (Silva et al., 2020).

O tratamento cirúrgico do aneurisma da aorta abdominal visa prevenir sua ruptura, prolongar a vida do paciente e 
aumentar a expectativa de vida. A chave é identificar os pacientes com maior chance de ruptura e menor chance de morrer por outras doenças, para que o tratamento cirúrgico possa trazer benefícios reais, que se caracterizam basicamente pelo aumento da expectativa de vida. Para pessoas com aneurismas maiores que $6 \mathrm{~cm}$, sintomáticos, ou que aumentaram de diâmetro em mais de $0,5 \mathrm{~cm}$ em 6 meses, os benefícios da cirurgia foram comprovados. No entanto, em alguns pacientes, as comorbidades relacionadas limitam os benefícios de uma cirurgia bem-sucedida (Becker et al., 2020).

\section{Conclusão}

O aneurisma de aorta abdominal é um distúrbio vascular que apresenta altas taxas de mortalidade, especialmente em pacientes idosos e que apresentam fatores de risco associados. Em geral, apresenta-se de forma assintomática e quando há presença de sintomas significa que há uma complicação e as taxas de sobrevivência diminuem de forma significativa.

Pacientes que apresentam fatores de risco, em especial, problemas cardiovasculares, dislipidemias, tabagismo, idade avançada, entre outros, possuem grande risco de rompimento da artéria, devido ao enfraquecimento da parede da aorta causada por esses fatores e/ou aumento da pressão dentro do vaso. A manifestação clínica de maior importância nesta doença é a ruptura do aneurisma, podendo na maioria dos casos ser letal.

O diagnóstico precoce realizado geralmente através de ultrassonografia é essencial para a diminuição dos óbitos por AAA. As taxas de sucesso das cirurgias eletivas de aneurisma de aorta abdominal são altas. Infelizmente ainda não existem terapias farmacológicas para esta patologia, sendo o procedimento cirúrgico a única forma de tratamento.

É necessária a utilização do diagnóstico precoce em pacientes que apresentem risco de desenvolver AAA, visto que o manejo cirúrgico eletivo tem permitido redução da mortalidade de forma considerável. Também são necessários estudos para o desenvolvimento de um tratamento farmacológico que se mostre eficaz em retardar a progressão e ruptura desses aneurismas.

\section{Referências}

Amaral, G. F. F., Fernandes, H. P. M., \& Alves, R. F. (2021). Aneurisma de aorta abdominal: uma análise clínico-epidemiológica do estado do Tocantins nos últimos 20 anos. Revista de Patologia do Tocantins, 8(1), 48-57.

Ascanio, C. R., Vázquez, I. P., \& Gainza, S. L. S. (2021). Aneurisma idiopático de aorta abdominal en una paciente de 4 años de edad. Medisan, 25(3), 715722.

Becker, M., Bonamigo, T. P., \& Faccini, F. P. (2020). Avaliação da mortalidade cirúrgica em aneurismas infra-renais da aorta abdominal. Jornal Vascular Brasileiro, 1(1), 15-21.

Silva, E. S., Hanaoka, M. H., Puech-Leão, P., \& de Tolosa, E. M. C. (2020). Relação morfológica entre o diâmetro, o colo proximal e distal dos aneurismas da aorta abdominal. Jornal Vascular Brasileiro, 3(2), 95-101.

Assis, J. A. C., \& Duque, M. A. (2020). Aneurisma de aorta abdominal, aspectos, patogenicidade, diagnóstico e terapia. Brazilian Journal of Development, 6(11), 92736-92749.

García, Y. D., Romero, M.M., Díaz, T.A., Leyva, L.M., Rey, J. C.O., \& Sicard, A.Y. (2021). Colangiopancreatografía retrógrada endoscópica en un paciente con gran aneurisma de la aorta abdominal. Revista Cubana de Medicina Militar, 50(1).

Gomes, G. R. G., D’Ornellas, M. C., \& Dotto, G. N. (2021). Medidas de aneurisma da aorta abdominal: modelos tridimensionais impressos e virtuais. Radiologia Brasileira, 54, 21-26.

Guzmán, M. D. C.H., Maruri, C. E.T., Mantilla, M. E.T., Seara, A.H., \& Cisneros, H.V. (2021). Complicaciones posquirúrgicas en pacientes con aneurisma de la aorta abdominal operados electivamente. Revista Cubana de Angiología y Cirugía Vascular, 22(2).

Menino, E. O.O., Libanio, G. C., Cardoso, L. B., \& Couto, B. R. G. M. (2020). Correção de aneurisma de aorta abdominal: Fatores de risco para a infecção do sítio cirúrgico e óbito. Brazilian Applied Science Review, 4(6), 3343-3354.

Novais, A. R. D. O., Brito, R. R. D., Yamanishi, V. F., de Jesus Gonçalves, A. N., Evangelista, J. M. P. L., Garcia, P. G. L., \& Batista, A. C. (2021). Aneurismas: qual a melhor terapêutica?. Brazilian Journal of Development, 7(9), 94661-94667.

Rojas, A. L. H., Martínez, M. M. M., \& Lopez, M.R. (2021). Diagnóstico precoz del aneurisma de la aorta abdominal asintomático en Pinar del Río. Revista de Ciencias Médicas de Pinar del Río, 25(3).

Santos, L. C. F., Paiva, M. A. F. D., Santana, M. V. L., Mendes, R., \& Tenório, P. P. (2021). Podemos adotar a dosagem sérica da Tenascina-C na avaliação prognóstica do aneurisma e dissecção da aorta?. Jornal Vascular Brasileiro, 20. 
Research, Society and Development, v. 11, n. 3, e15211326251, 2022

(CC BY 4.0) | ISSN 2525-3409 | DOI: http://dx.doi.org/10.33448/rsd-v11i3.26251

Silerio, I. G. C. (2021). Tratamiento farmacológico de aneurismas de la aorta abdominal. Expresiones Médicas, 9(1), 45-53.

Siqueira, N. L. A. D. S. (2021). Avaliação da influência da anatomia dos aneurismas de aorta abdominal infra-renal nos eventos intra-operatórios e nos desfechos clínicos no pós-operatório na Unidade de Terapia Intensiva após cirurgia endovascular. (Dissertação).

Touma, J., Bosse, C., Marzelle, J., Desgranges, P., \& Cochennec, F. (2021). Rupturas de aneurisma de la aorta abdominal: técnicas específicas. EMC-Cirugía General, 21(1), 1-26.

Trintinalha, P. D. O., Sarquis, L. M., Michaelis, W., Santos, A. L., Yokoyama, R. A., Miguel, M. T., \& Oliveira, M. S. D. (2021). Derivação axilo-ilíaco para proteção de rim transplantado durante a correção de aneurisma de aorta abdominal: relato de caso. Jornal Vascular Brasileiro, 20.

Zayas, S. L. Ranoni, Á. D.B., Ibarra, F. C. C.1., Gueyraud, L. T.C, \& Lesme, G. L.E. (2021). Reparación de aneurisma de aorta torácica con compromiso de vasos del cuello en paciente con sífilis terciaria. Reporte de caso. Rev. Nac.(Itauguá), 88-98. 